\title{
Acupuntura en un servicio hospitalario en Argentina: experiencias y perspectivas de los usuarios*
}

\author{
Betina Freidin ${ }^{1}$ \\ Rosana Abrutzky²
}

FREIDIN, B.; ABRUTZKY, R. Acupuncture in a hospital setting in Argentina: users' experiences and perspectives. Interface - Comunic., Saude, Educ., v.15, n.37, p.505-18, abr./jun. 2011.

We investigate the experiences and perspectives of users of a public hospitalbased service of acupuncture located in the Northwest region of Argentina. In 2005 we interviewed 18 patients. They use acupuncture for chronic conditions, mostly to alleviate pain-- although several extended its use over time to treat other health problems-because of the limited efficacy experienced with biomedicine and the adverse effects of medications, or to avoid surgical interventions. They incorporate acupuncture into pluralistic health care strategies, which in addition to biomedicine typically include other unconventional medicines. The hospital context facilitates the use of an alternative medicine, and some patients find in the service guidance to "negotiate" treatments with conventional physicians. The organizational efficiency and the doctors' commitment to their work were highly valued. Due to the increasing demand for treatments in a context of scarce resources, patients worry that the availability of appointments and doctor-patient interactions may be affected.

Keywords: Complementary and alternative medicine. Integrative medicine. Acupuncture. Public health sector.
Indagamos las experiencias y perspectivas de los usuarios de un servicio hospitalario público de acupuntura del noroeste argentino. Basamos el análisis en 18 entrevistas semi-estructuradas realizadas en el año 2005. Los entrevistados recurren a la acupuntura por dolencias crónicas, especialmente como paliativo del dolor, aunque varios extienden su uso para otros problemas de salud, frente a la ineficacia y/o efectos adversos de los tratamientos biomédicos, o para evitar cirugías menores. Incorporan la acupuntura a estrategias pluralistas de cuidado que junto con la biomedicina incluyen típicamente otras medicinas no convencionales. El contexto hospitalario facilita que prueben una medicina foránea. Varios usuarios encuentran el servicio de asesoramiento para "negociar" los tratamientos con sus médicos convencionales. Destacan la eficiencia organizativa del servicio y el compromiso de los médicos. Ante la demanda creciente de atención en un contexto de escasez de recursos advierten que ésta puede afectar la disponibilidad de turnos y la interacción médico-paciente.

Palabras clave: Medicina alternativa y complementaria. Integración. Acupuntura. Servicios de salud públicos.
"Este estudio fue financiado por la Universidad Brandeis (Janes's Travel Grant summer 2005) y los aspectos éticos del mismo revisados y aprobados por el Institutional Review Board de dicha universidad. Una versión anterior de este trabajo,

"¿Medicina alternativa y complementaria para todos? La experiencia de los usuarios de un servicio hospitalario de acupuntura en Argentina", fue presentada en el XXVII Congreso de la Asociación

Latinoamericana de Sociología (ALAS),

Buenos Aires del 31 de agosto al 4 de septiembre de 2009.

${ }^{1}$ Consejo Nacional de Investigaciones Científicas

y Técnicas, Instituto de

Investigaciones Gino

Germani, Facultad

de Ciencias Sociales,

Universidad de Buenos Aires (UBA). Uriburu 950, Piso 6, CABA, Argentina. freidinbetina@gmail.com 2 Instituto de

Investigaciones Gino Germani, Facultad de Ciencias Sociales, UBA. 


\section{Introducción}

La literatura documenta que las medicinas alternativas y complementarias (MACs) son ampliamente utilizadas en Argentina fuera del sistema de salud oficial; sin embargo, poco sabemos sobre las perspectivas y experiencias de los usuarios cuando éstas ingresan al sector público. Las investigaciones sobre el uso de medicinas no convencionales entre los sectores sociales más desfavorecidos se han centrado en el autocuidado, la medicina popular o tradicional y la religiosa (Idoyaga Molina, Luxardo, 2005; Idoyaga Molina, 2002; Viturro, 1998, entre otros). En este artículo indagamos la utilización de la acupuntura entre dichos sectores en un servicio hospitalario. Se trata de una MAC asociada al consumo terapéutico de la clase media y alta por factores culturales y económicos (Freidin, Abrutzky, 2010; Carozzi, 2000). Debido al ingreso, aunque muy lento, de la acupuntura en el sistema de salud oficial, especialmente en el ámbito público, el propósito de este artículo es dar visibilidad a la experiencia de la utilización de acupuntura entre usuarios "atípicos".

\section{Contexto institucional y legal de la acupuntura en Argentina}

La Organización Mundial de la Salud (OMS) ha documentado que la creciente difusión y utilización de MACs es un fenómeno global y ha ejercido un rol activo para incorporarlas en los sistemas de salud en los distintos contextos nacionales, atendiendo a sus realidades socio-culturales y siguiendo criterios de uso racional y seguro (Freidin, Abrutzky, 2010). En el ámbito regional, un Grupo de Trabajo de la OPS y la OMS en el año 1999 recomendó promover actividades que apoyen el mejor conocimiento de las MACs con el fin de identificar estrategias que contribuyan a la organización y prestación de sistemas de salud integrales y culturalmente apropiados en las Américas (OPS, 2002). Pese a estos esfuerzos, y a diferencia de lo que ocurre en varios países desarrollados, las iniciativas de los gobiernos para integrarlas en los sistemas de salud en los países de la región, en el nivel de la atención primaria, han sido escasas. Pocos han desarrollaron políticas nacionales en la materia, destacándose entre ellos Cuba, Perú, y Bolivia (OPS, 2002). En Argentina la integración de medicinas no convencionales en el sistema de salud oficial ha sido excepcional, siendo la acupuntura la que en los últimos años ha avanzado en esta dirección (World Health Organization - WHO, 2005; Organización Panamericana de la Salud, 2002).

Introducida en círculos médicos durante los '50, la acupuntura es una de las pocas MACs reguladas en la Argentina. Aunque no existe una ley nacional sobre el ejercicio de la acupuntura, en el año 2001 el Ministerio de Salud de la Nación estableció mediante la Resolución 997 que la acupuntura es un "acto médico" que sólo podía ser realizado por los profesionales de la salud regidos por la Ley 24.317. Las autoridades sanitarias y Colegios Médicos de la mayoría de las provincias convalidaron esta normativa (WHO, 2005). En el año 2008, la Resolución Ministerial 859 amplió la autorización a kinesiólogos y fisioterapeutas. Con estas medidas se favoreció la incorporación de la acupuntura en el sistema de salud tanto en establecimientos privados como públicos, aunque dicha integración ha sido muy lenta y presenta una gran disparidad provincial (Freidin, Abrutzky, 2010). El apoyo del Ministerio ha convergido con algunas pocas políticas de salud provinciales que han propiciado la incorporación de la acupuntura en su esquema asistencial público, tanto en hospitales como en centros de salud comunitarios. Este es el caso de la provincia en donde realizamos el estudio cuyos resultados aquí presentamos. 


\section{Objetivos y metodología}

${ }^{3}$ Las entrevistas con los usuarios del servicio fueron parte de un estudio más amplio en el que la primera autora entrevistó al personal médico del mismo y a médicos acupuntores de otras localidades.

${ }^{4} \mathrm{Al}$ inicio de la entrevistas entregamos a cada entrevistado/a una nota de consentimiento informado que garantizaba el anonimato y la confidencialidad en el análisis de la información proporcionada. Para garantizar el anonimato del personal médico del servicio omitimos identificar la ciudad en el que se encuentra.
El objetivo general del artículo es indagar las experiencias y perspectivas de los usuarios de un servicio hospitalario de acupuntura en una ciudad del noroeste del país ${ }^{3}$. Más específicamente indagamos: 1) Las razones por las que utilizan acupuntura, y las experiencias con los tratamientos y sus proveedores en un contexto hospitalario; 2) Cómo incorporan la acupuntura en sus estrategias de cuidado de la salud; 3) Cómo caracterizan al servicio de acupuntura y lo distinguen de otros efectores de salud, especialmente públicos; y 4) Cuáles son, desde la perspectiva de los usuarios, las dificultades que enfrenta el servicio ante la creciente demanda de tratamientos.

En junio de 2005 concurrimos al servicio durante 10 días lo que nos permitió observar su funcionamiento, especialmente la interacción entre los pacientes, y entre ellos y el staff médico y no médico. Para entrevistar a los pacientes solicitamos a los médicos que los invitaran a participar del estudio, estando la investigadora a cargo del estudio presente durante dicha solicitud para explicar su propósito, que el mismo no había sido solicitado por el servicio ni por las autoridades del hospital, y para asegurar el carácter voluntario y anónimo de la participación ${ }^{4}$. Realizamos entrevistas cualitativas semi-estructuradas con 18 pacientes. Buscamos cierta variabilidad etárea y de género entre ellos, teniendo en cuenta que según las estadísticas del servicio el $76 \%$ de los usuarios son mujeres y el $75 \%$ de las consultas corresponde a personas de entre 40 y 79 años. Con el número de entrevistas realizadas logramos saturar las dimensiones de análisis desarrolladas en el presente artículo. Realizamos las entrevistas en un café adyacente al hospital y en un consultorio fuera del servicio que nos fue facilitado para tal fin. Fuera del horario de atención, entrevistamos a los médicos. Si bien en este artículo nos centramos en las perspectivas de los pacientes, dichas entrevistas al igual que las observaciones realizadas en el lugar nos permitieron contextualizar las visiones y experiencias de los usuarios, además de brindarnos información sobre el funcionamiento del servicio. Las entrevistas fueron grabadas digitalmente y desgrabadas verbatim. Las analizamos siguiendo la estrategia del análisis temático cualitativo asistido por el programa ATLAS.ti.

\section{El servicio de acupuntura}

El servicio opera desde hace varios años en un hospital público en una ciudad del Noreste argentino, y está afiliado al departamento de Clínica Médica. La mayoría de los usuarios residen en la ciudad, aunque concurren personas de otras localidades y provincias limítrofes. El hospital atiende a una población de bajos recursos, en términos de ingresos y de educación formal. Sin embargo, el servicio de acupuntura debido a la creciente difusión de sus actividades a través de la prensa local y del boca en boca atrajo también a usuarios de sectores medios. Al igual que lo ocurrido en otras localidades, tras la severa crisis socioeconómica del 2001-2002 el servicio comenzó a recibir la demanda de atención de sectores medios empobrecidos que recurrieron al hospital público frente a situaciones de desempleo de largo plazo o de gran deterioro del poder adquisitivo de sus ingresos. Según información oficial de la provincia publicada en el año 2006, basada en datos del INDEC del 2001, el 48, 22\% de la población no tenía cobertura médica de obra social o plan médico. Esta situación se ve reflejada en nuestras entrevistas del 2005: de los 18 entrevistados, 8 accedían a servicios de salud únicamente en el sector público (hospitales y centros de atención primaria), 
7 tenían cobertura a través de obras sociales nacionales o de la provincia, y sólo tres contaban con cobertura de medicina prepaga.

El servicio funciona de lunes a viernes por la mañana; cuando realizamos el trabajo de campo trabajaban en él cuatro médicos con dedicación parcial, y otros dos profesionales adscriptos concurrían una vez a la semana. Opera como consultorio externo, con un sistema de turnos que incorpora paulatinamente nuevos pacientes. Se otorgan aproximadamente 56 turnos por día, cada uno de 30 minutos. Las nuevas admisiones se realizan dos veces por mes. Tras la entrevista de admisión con el director del servicio, se estructura para cada paciente un cronograma de turnos según su dolencia y gravedad. Si bien una vez admitidos los pacientes ven asegurada la continuidad del tratamiento (que inclusive puede tener una frecuencia semanal de tres sesiones), en la práctica ellos se ven limitados para realizar consultas preventivas, ya que cuando la situación del paciente mejora, el staff va espaciando sus turnos de manera de poder dejar horarios libres para nuevos pacientes.

El servicio atiende gratuitamente a los pacientes. Esto no fue siempre así ya que cuando los hospitales públicos fueron arancelados, se cobraba un bono de \$ 3 por sesión, al igual que en los restantes servicios. Los entrevistados más antiguos no resaltaron este factor como un elemento decisivo en la continuidad del tratamiento, aunque veían como muy positivo que actualmente fuera gratuito, especialmente si tenían un costo considerable de traslado y concurrían al servicio varias veces por semana, y estaban desocupados o tenían ingresos muy bajos.

\section{Características de los entrevistados}

Los pacientes que entrevistamos tienen entre 26 y 73 años (la edad promedio es de 50.8 años). 13 son mujeres y 5 son varones. Su nivel de educación formal es variado: 2 no completaron la escuela primaria, 6 completaron estudios primarios y/o iniciaron el secundario, 3 completaron dicho ciclo, 5 iniciaron estudios universitarios o terciarios que no completaron, y 2 completaron carreras universitarias. En cuanto al perfil laboral, 2 de los entrevistados son profesionales en actividad, 4 perciben jubilaciones o pensiones, 2 están desocupados, 3 mujeres se definen como amas de casa, y los restantes 7 desempeñan ocupaciones u oficios, en relación de dependencia o por cuenta propia, en tareas manuales de baja calificación (tejedora, modista, remisero, o venta de ropa).

La antigüedad de los entrevistados como usuarios del servicio es muy dispar (entre 2 sesiones y 14 años). Seis entrevistados fueron derivados al tratamiento de acupuntura por otros médicos, entre ellos tres profesionales que conocían o practicaban acupuntura. Los médicos del servicio destacaron que pese a que el mismo había sido oficializado desde hacía varios años por las autoridades sanitarias de la provincia y del hospital, después de haber tenido un estatus inicial de práctica experimental, este hecho no resultó en un aumento de derivaciones formales por parte de colegas de otros servicios. De hecho, la mayoría de los pacientes llegó al servicio a través de sus redes personales, por recomendación de familiares, vecinos y conocidos que eran pacientes del mismo. Un entrevistado se enteró de su existencia por los medios de comunicación locales, mientras que otro recurrió al servicio aconsejado por una sanadora espiritual (ver más abajo, cuando describimos el uso de otras medicinas no convencionales por parte de los pacientes).

\section{Los motivos de consulta y las razones para utilizar acupuntura}

Los motivos de salud por los cuales los pacientes realizaron la primera consulta son variados, aunque predominan los problemas crónicos. Más de la mitad de los entrevistados buscaban paliar dolores crónicos producidos por artrosis, artritis, y problemas de columna. Todos ellos habían utilizado tratamientos paliativos biomédicos, y en algunos casos otras MACs, con los que experimentaron limitada o nula eficacia. Los entrevistados mencionaron en varios casos además de las dolencias 
físicas las consecuencias anímicas que les producía el dolor cónico (falta de voluntad y desánimo, mal humor, y depresión). Asimismo, destacaron los efectos adversos de la medicación farmacológica que se prescribe para este tipo de dolencias (ej. desordenes digestivos). Evitar una intervención quirúrgica (por cálculos renales, varices, o túnel carpiano) también motivó la búsqueda de una alternativa terapéutica no convencional.

Quienes recurrieron a la primera consulta para tratar problemas alérgicos o respiratorios (asma) mencionaron especialmente los efectos negativos del consumo de corticoides como un elemento determinante a la hora de tomar la decisión de probar una terapia no convencional. En general los efectos iatrogénicos de la medicación farmacológica fueron mencionados por los entrevistados como una de las razones que los llevaron a la acupuntura, y consecuentemente, como veremos, consideraron como un gran beneficio del tratamiento poder disminuir o abandonar la utilización de fármacos. En tres casos los padecimientos psíquicos (stress, depresión, insomnio) motivaron la primera consulta, combinados en un caso con neuralgias, en otro con problemas circulatorios, y en un tercero con dolores articulares crónicos.

\section{La eficacia del tratamiento desde la experiencia de los pacientes}

Excepto en un caso, los entrevistados experimentaron una alta eficacia con el tratamiento de acupuntura. Algunos enfatizaron el hecho que para su dolencia crónica no existía cura pero que la acupuntura les proporcionó un gran alivio, disminución o desaparición de los síntomas y les permitió mejorar notoriamente su calidad de vida y funcionalidad en la vida diaria. Como explica una paciente de 26 años que padecía artrosis desde la adolescencia:

\footnotetext{
"Me cansé de probar cosas que no funcionaron y al encontrar algo que funciona, uno eso lo resalta. [...] y uno busca el alivio, el alivio del dolor porque eso es la enfermedad en sí, un alivio al dolor terrible en el hueso y uno busca alivio para ese dolor y con la acupuntura lo conseguí, no del todo, o sea, no me sacó todo, todo el dolor, pero me sacó, me alivió bastante, bastante. [...] Esto funciona, por fin hay algo que funciona, encuentro algo que funciona, que me ayuda". (E07)
}

El aumento en la movilidad y la disminución del dolor fueron motivos suficientes para continuar con el tratamiento, sobre todo cuando los tratamientos convencionales mostraron limitada o nula eficacia. Así lo destaca una paciente de 70 años que sufre de artrosis:

\footnotetext{
"El doctor me dijo que como antes no voy a hacer las cosas, con este problema no, yo cocino algunas cositas, pero limpiar la casa, limpiar el piso, lavar ropa grande eso no puedo, no, ropa chica sí. [...] yo, como le dije, no podía caminar, en cambio ahora tengo una mejoría de $100 \% "$. (E17)
}

En todos los relatos, los pacientes resaltaron que la acupuntura no produzca los efectos secundarios de los tratamientos farmacológicos, enfatizando el carácter de "natural" e inocuo de este tipo de medicina. "Es lo único sano, es una cosa natural, digamos, ¿qué puede afectar?", comenta una paciente de 63 años que padece de depresión y artrosis. Otra entrevistada de 46 años que padecía la misma enfermedad articular también se explaya sobre la ausencia de efectos adversos en el tratamiento, lo que le parece "milagroso" frente a los tratamientos convencionales:

“La acupuntura me hizo pasar el dolor y dejar los medicamentos, que para mí eso fue milagroso, que dejés de tomar medicamentos es, si yo vivía enferma del estómago, de los nervios, todo, y era de la cantidad de remedios que tomaba". (E11) 
Otros entrevistados destacaron que la acupuntura es una opción terapéutica no invasiva y optan por probarla frente a la prescripción de una intervención quirúrgica. En un caso, el propio médico de cabecera de una mujer de avanzada edad que sufría túnel carpiano le recomendó el tratamiento para evitar el riesgo de una cirugía. Pero, en general, son los mismos pacientes los que buscan segundas opiniones médicas y tratamientos menos agresivos, aconsejados por familiares y conocidos, y/o médicos allegados con conocimiento de acupuntura. Una paciente de 41 años que tenía cálculos renales y a la que el urólogo de otro hospital le había prescripto una operación convencional (por problemas económicos la paciente no podía solventar a una litotricia) buscó otras opiniones profesionales:

“El urólogo vio los estudios y ahí nomás me quería operar, pero yo no me conformo con una primera opinión. Comencé a consultar con amigas que son estudiantes de medicina y he empezado a preguntar en los centros de salud". (E08)

Su madre le recomendó el servicio de acupuntura. Después de dos años de tratamiento observó que no sólo tenía efecto analgésico, sino que los cálculos se habían reducido en tamaño y cantidad:

"Yo pienso que la operación sería una última instancia, creo que un médico tiene que ver primero cualquiera otra posibilidad antes de operar. [...] Porque no es fácil que te metan un cuchillo en tu cuerpo". (E08)

Además de una mejoría para el problema específico por el que iniciaron el tratamiento, varios entrevistados resaltaron "beneficios adicionales", tales como mejora del ánimo y disminución de la ansiedad, mejor humor, y una sensación general de bienestar. Los más entusiastas utilizaron expresiones tales como "es mi salvación", "soy otra persona", "es una bendición", "me ayuda a llevar una vida digna" para describir la eficacia del tratamiento. Sólo un entrevistado no percibió mejora alguna atribuible a la acupuntura para su problema de salud, pero igualmente continuó el tratamiento ya que los médicos del servicio le habían anticipado que su caso no era fácil (rehabilitación por parálisis facial parcial).

La mayoría de los entrevistados expandió el uso de la acupuntura a dolencias diferentes de aquella por la cual realizó la primera consulta. En algunos casos, durante la entrevista de admisión se manifiesta que tienen otras dolencias para las cuales la acupuntura podría proporcionar alivio o cura. En otros casos, los pacientes mismos al familiarizarse con el tratamiento y comprobar su eficacia les piden a los profesionales que los ayuden a solucionar otros problemas de salud. La mayoría de los entrevistados que expandieron el uso de la acupuntura lo hicieron para problemas psíquicos/anímicos y/o gastrointestinales. Dolores menstruales, incontinencia urinaria, disminución de peso, várices y sinusitis son otras condiciones para los cuales los pacientes ampliaron el uso de la acupuntura.

\section{Las prácticas de integración terapéutica de los pacientes y el rol facilitador del contexto médico-hospitalario}

La totalidad de los entrevistados utilizan la acupuntura de manera complementaria a la biomedicina. Esto es así porque combinan el tratamiento de acupuntura con los de la medicina convencional para tratar un mismo problema de salud, y/o porque continúan visitando a médicos especialistas o generalistas para tratar otras dolencias o por consultas preventivas (chequeos ginecológicos, de colesterol, etc.). Así como observamos el uso extendido a lo largo del tiempo de la acupuntura para tratar varios problemas de salud, algunos entrevistados, sin embargo, utilizan la acupuntura en forma limitada porque encuentran respuestas satisfactorias en la biomedicina para otras dolencias. Una paciente de 70 años que recurrió a la acupuntura para tratar un cuadro de estrés da cuenta del este uso restringido: 
“No la he usado para otros problemas de salud, sólo para la parte anímica. Y no, porque otros problemas, digamos, de gastroenterología que tengo, me atiende el gastroenterólogo y ando bien con la medicación". (E10)

Una paciente de 49 años recurre a la acupuntura sólo para el alivio de dolor crónico de columna:

"Yo tengo mi ginecóloga, tengo mi cardiólogo, [el hecho de estar haciendo acupuntura no implica] decir que no voy a ir más [a verlos]". (E02)

El hecho de que los tratamientos de acupuntura sean brindados por profesionales médicos facilita las prácticas de complementación de recursos terapéuticos generalmente iniciada por los propios pacientes (como ya lo señalamos, muy pocos llegan al servicio por una derivación médica formal). Los entrevistados comentan que no encuentran dificultad en compartir con su médico especialista o generalista que están haciendo acupuntura, encontrando creciente aceptación de su parte tanto de la acupuntura como del servicio. El contexto institucional facilita las estrategias de integración terapéutica de los pacientes, ya que varios de ellos se tratan con otros profesionales del mismo hospital. Incluso si los médicos no pertenecen al establecimiento, existe la posibilidad de articular estrategias de tratamiento. Los entrevistados ven esta posibilidad de coordinación como una gran ventaja para el cuidado de su salud. Una entrevistada de 26 años nos relata su experiencia:

“[La medicación para la artrosis] me trajo problemas al estómago [...].Después empecé a ver al Dr. $\mathrm{X}$, queríamos que trabajen en conjunto con mi reumatólogo, que se conocen entre ellos, y yo le comenté a mi reumatólogo que estaba yendo a acupuntura y me dijo "está bien, si a vos te hace bien seguí", y bueno, y ahí empezamos a bajar las dosis de los medicamentos, algunos los sacamos". (E07)

Los entrevistados consideran además que los médicos acupuntores pueden guiarlos en cuanto a la atención biomédica que reciben en otros servicios, lo que los ayuda a ser más demandantes cuando interactúan con sus médicos "ortodoxos". Como lo relata una entrevistada, en el servicio los médicos contemplan el cuadro general del paciente, superando la visión reduccionista de los especialistas. Esta visión integradora se ve facilitada por la formación en clínica médica o en medicina familiar de los médicos, además de su especialización en acupuntura que es una medicina holística. Una entrevistada de 41 años que sufría de varios problemas de salud crónicos (anemia por mioma uterino, cálculos renales y trastornos anímicos) y por los cuales consultaba a diferentes especialistas ortodoxos, valoró especialmente el enfoque integrador y la orientación que encontró en el servicio:

\footnotetext{
“No sé si será la provincia o qué, acá cada médico está en lo suyo nada más, no van un poquito más allá. [En cambio en el servicio] te preguntan y por ahí te orientan, "¿cómo no le han hecho este estudio?, ¿cómo no le han hecho este otro?", entonces vos vas y le preguntás al médico de vuelta, "doctor, ¿por qué no me ha hecho tal cosa?" - "ah, no, ah, no me di cuenta, se me ha pasado, o no es necesario" - te dicen - . Pero uno ya va más o menos sabiendo, y siempre que me he hecho un estudio, por más que sea de sangre, yo siempre lo traigo y lo hago ver al doctor, acá en acupuntura, y él va anotando y registrando en la historia clínica". (E08)
}

De este modo los entrevistados consideran que pueden ejercer más control sobre el cuidado de su salud. Cuando interactúan con los médicos convencionales, este margen de maniobra se traduce en la percepción de una mayor capacidad para demandar y negociar los tratamientos. Una entrevistada de 63 años que utilizaba acupuntura para sobrellevar el stress y la depresión, y que había experimentado importantes efectos adversos con la medicación psiquiátrica, comenta: 


\begin{abstract}
“La medicación psiquiátrica me hace mal al estómago, no la tolero, entonces el psiquiatra ya no sabía que es lo que me iba a dar. Le digo, "no me dé la receta porque no lo voy a comprar". [...] Un día le digo, "doctor yo le voy a contar algo no sé si se va a reír o qué me va a decir [...] yo me estoy haciendo acupuntura", "no me digas" [...] le digo - "no se gaste en conseguirme pastillas" [...] "querés que te diga una cosa, seguí con la acupuntura -me dice-me parece muy bien" ". (E10)
\end{abstract}

Además del uso complementario de la acupuntura y la biomedicina, la mayoría de los entrevistados (15) utilizaban, o habían utilizado anteriormente, otras medicinas no convencionales en sus estrategias de cuidado de la salud. Entre ellas, la homeopatía, la medicina tradicional local, prácticas de autocuidado, y la medicina religiosa. Utilizan múltiples modalidades terapéuticas, sucesiva o simultáneamente, según su disponibilidad y accesibilidad, familiaridad, y siguiendo su criterio - y la de su entorno social - sobre el uso seguro y apropiado de las mismas. Así nos lo explicó una paciente muy conocedora de la herboristería local, que concurría al servicio desde hacía varios años para el alivio del dolor de columna:

“En vez de hacer bien los remedios de tanto tomar ya se intoxica el cuerpo [...] yo no soy de esas de tomar mucho remedios. [...] como yo me he criado en el campo [...] [donde] sacan todo de la parte natural de las plantas [...] uno a veces las conoce a las plantas y puede tomarse un té, por ejemplo [para] un dolor de estómago puede tomar un té de poleo [pero] no tomar de más [...] hay que ser cauteloso en todas las cosas". (E15)

Y también nos comentó una paciente de 26 años sobre sus plegarias para sobrellevar crisis asmáticas:

\footnotetext{
"Cuando tengo estas crisis de asma [le pido] por favor San Expedito que pueda respirar, porque es como que realmente me ahogo. [...] Me ayuda, sí [...] me alivia". (E05)
}

El uso complementario de medicinas no convencionales para el tratamiento de la misma o diversas dolencias está documentado en estudios extranjeros (Barnes, Bloom, Nahin, 2008; Astin, 1998; Sharma, 1996, entre otros) y nacionales (Freidin, Abrutzky, 2010; Idoyaga Molina, Luxardo, 2005; Idoyaga Molina, 2002). Nuestro trabajo corrobora el pluralismo terapéutico entre los usuarios del servicio en un área geográfica en la que estas medicinas coexisten con la medicina convencional en el sistema etnomédico local (Idoyaga Molina, 2002). Si bien la mayoría de los entrevistados incorporan la acupuntura a un patrón ya pluralista de cuidado de la salud, en general, se trata de una medicina foránea sobre la cual tenían poco conocimiento, les era culturalmente distante, y que les generaba ciertos temores ("miedo a los pinchazos"). Sin embargo, el contexto hospitalario en el que se brindan los tratamientos les inspiró confianza y aumentó la credibilidad en la acupuntura; y las experiencias positivas de personas conocidas, además del acceso gratuito, hicieron que se animaran a probarla y a testear su eficacia.

\title{
¿Cómo evalúan los entrevistados al servicio de acupuntura dentro del sistema de atención pública de la salud?
}

\section{Confort y eficiencia organizativa}

En comparación con otros servicios públicos de salud, o de la atención brindada por el PAMI, los entrevistados destacaron como aspectos positivos la organización del servicio y el hecho que los tratamientos se brinden en un sitio confortable. Así, al evaluar la calidad de la atención del servicio 
de acupuntura destacaron como factores positivos diferenciales el hecho que se cumplan los horarios programados de los turnos, la posibilidad de recibir los tratamientos en un lugar con calefacción, ventiladores, y con cortinas para mantener la privacidad de los pacientes mientras son atendidos en boxes individuales, así como la higiene del lugar:

\footnotetext{
"Acá yo me siento bien porque es un servicio público y en la Argentina no tenemos grandes cosas públicas, estamos acostumbrados a que a veces nos atienden en el pasillo [...]. Por ejemplo me toco ir a la guardia del [hospital X], que es un entidad publica de acá; fui a la mañana, me llevaron en una ambulancia porque estaba deshidratada y me tuvieron en una silla hasta las once de la noche, no me habían registrado en los libros [...]. Entonces venir acá y tener la camilla para mí es maravilloso [...].O por ejemplo, vamos a un sanatorio que nos corresponde por PAMI y nos atienden de favor. Entonces yo acá estoy muy cómoda, me siento muy cómoda". (E10)
}

Aunque las cortinas de los boxes para los tratamientos garantizan la privacidad visual, desde cada box se escucha lo que se habla en los aledaños y en el pasillo dentro del servicio que sirve como sala de espera. Para nuestra sorpresa, el hecho que se escuchen las conversaciones no fue visto como un problema. Algunos pacientes dijeron que no les molestaba o que no les prestaban atención porque se relajaban durante la sesión de acupuntura; en otros casos, incluso, lo veían como un agregado aprovechable o divertido a la situación de tratamiento. La socialización de las conversaciones entre los médicos y los pacientes lleva además a que éstos puedan adquirir información adicional acerca de los beneficios terapéuticos de la acupuntura que no eran de su conocimiento. Los entrevistados destacaron esta situación como una importante fuente de información para sus estrategias de complementación terapéutica.

Sin embargo, lo más valorado por los pacientes como característica extraordinaria del servicio fue la eficiente organización del sistema de turnos, y la puntualidad y compromiso por parte del personal médico para con los usuarios. Los turnos se respetan, el personal médico cumple su horario, y los boxes siempre están ocupados:

"La gente que viene consigue turno, es bien atendida porque los médicos son fabulosos, se quedan después de hora, es la 1 de la tarde y si ellos tienen gente te atienden. Atienden a muchísima gente por día [...] si te levantas de la camilla, ya esta otro paciente. O sea, ellos sí que trabajan". (E11)

\footnotetext{
${ }^{5}$ Numerosos estudios sobre satisfacción de los usuarios con los servicios de salud públicos documentan estas deficiencias (Cerrutti, Freidin, 2004; Bianconi et al., 2000; Ramos, Pantelides, 1990; Llovet, 1984, entre otros)
}

En contraste con estas condiciones, los entrevistados relataron experiencias frustrantes en otros servicios hospitalarios, o en la atención por obras sociales y el PAMI, en las cuales enfrentan esperas larguísimas para conseguir un turno, los horarios no se cumplen o los turnos se cancelan sin previo aviso ${ }^{5}$. El cumplimiento del horario de atención y de los turnos en el servicio fue visto como un gesto de respeto y cuidado del paciente. Los médicos son concientes de la importancia que los pacientes dan a este aspecto de la atención en un contexto hospitalario, y se esfuerzan por mantener esta característica distintiva del servicio. Al respecto nos comentó el director: 
“El paciente está acostumbrado a un trato despersonalizado, [y en el Servicio] se va con un papelito que dice lo esperamos el miércoles a la mañana o el jueves a tal hora, y no tiene que hacer cola, como si fuera una consulta privada con su turno esperándolo. Y comprueba que efectivamente lo estamos esperando a esa hora, y comprueba que a las 7:30 que empieza la atención estamos acá [...]. Yo a veces los he escuchado [en la sala de espera] "¿Usted ha visto alguna vez alguien [el Director] que venga tempranito y sea el ultimo en irse?". Ellos sienten que se los trata de otra manera [...]. Creo que eso los contiene, los hace sentir bien. Y a eso súmale que es gratis y que encuentran una solución que no venían encontrando en la medicina convencional".

\section{La calidad de la atención médica:}

\section{Competencia técnica e interaccional, y pasión por la tarea}

Además del hecho que se respeten los turnos, los entrevistados valoran muy especialmente el trato que reciben de los médicos en el servicio. Ponen de relieve la atención personalizada y centrada en el paciente. En este sentido, además de la competencia técnica de los profesionales, aluden a la competencia interaccional y a sus cualidades personales. Destacan que los médicos se interesan por los pacientes, hablan con ellos, son amables y están dispuestos para atenderlos, características que desde la perspectiva de los entrevistados también distingue al personal del servicio del de otros efectores públicos. Una entrevistada resaló que la dimensión afectiva de la relación médico-paciente tiene en sí misma efectos terapéuticos:

\footnotetext{
"Cuando Ud. encuentra un médico amable que habla con Ud., que la trata como a Ud. le gusta, que está preguntándole todo, es algo que a todos nos gusta. Porque a veces a los pacientes no tanto les hace bien los medicamentos como la charla del médico". (E03)
}

Una paciente que se había atendido en forma privada con uno de los doctores, incluso comentó que "es la misma atención que tiene en su consultorio" (E10), y otra nos contó que si durante el tratamiento siente alguna incomodidad con las agujas, "viene cualquiera, no es sólo el médico que te ha atendido, están todos ahí para atenderte" (E11).

Cabe aclarar que como contactamos a los entrevistados a través del servicio este hecho puede introducir un sesgo positivo en sus opiniones sobre el mismo. Además de la competencia técnica y para relacionarse con los pacientes, los entrevistados destacaron la vocación, compromiso, y "pasión" por su trabajo. Así algunos se refirieron a ellos como profesionales "enamorados de su trabajo" o "locos por la acupuntura". Un entrevistado se explayó al respecto:

\footnotetext{
"Ese bohemio del profesional que quiere con el esfuerzo de su estudio sacar a alguien de un problema [...] [el jefe del servicio] está rodeado de gente de su calaña, del palo de él, sí, sí, están todos locos, locos por la acupuntura, locos por imponer su verdad [E: sí, son muy comprometidos] - Sí, exactamente, esa es la palabra, y se nota, lo nota la gente, lo nota en la piel". (E16)
}

\section{Dificultades para atender a la creciente demanda y cómo afectan a los usuarios del servicio: la escasez de recursos y el ritmo hospitalario}

Ante la pregunta sobre qué cosas cambiarían o se podrían mejorar, los entrevistados hicieron referencia a varios aspectos estructurales del servicio: la necesidad de contar con mayor espacio físico, más boxes para los tratamientos, y más personal médico rentado. Estos cambios permitirían absorber la creciente demanda de tratamientos, estimulada tanto por la difusión en los medios de prensa locales de las actividades del servicio como por la gran publicidad "del boca en boca" que hacen propios usuarios.

Si bien la difusión de información sobre la existencia del servicio es vista como algo muy positivo ya que brinda la posibilidad a mucha gente de acceder a una medicina que de otro modo no estaría a su 
alcance, por otro lado, los usuarios advierten que la demanda masiva presenta varias dificultades. La escasez de recursos materiales y humanos en un contexto de sobre-demanda de tratamientos incide en la cantidad de turnos disponibles y en su distribución entre nuevos ingresantes y los pacientes ya incorporados al servicio.

En primer lugar, los entrevistados mencionan las crecientes dificultad de acceder a la primera entrevista de admisión, "[Conseguir turno] últimamente no es muy fácil porque está, está viniendo mucha gente, muchísima gente, gente que está esperando un mes" (E15). En segundo lugar, el ingreso de nuevos pacientes limita la cantidad de turnos disponibles para aquellos que ya son pacientes regulares del servicio y el tiempo que disponen los médicos para atender a cada paciente.

En este contexto restrictivo, los entrevistados refirieron a la existencia de una regla implícita de solidaridad consistente en no "abusar" en la solicitud de turnos, de no faltar a las sesiones programadas, y de ser puntuales. Inclusive algunos comentaron que se contenían de solicitar turnos a menos que sus síntomas fuesen muy serios, o no pudieran soportar los dolores. Por ejemplo, una paciente con cálculos renales a veces tomaba tés medicinales como un sustituto para controlar el dolor, mientras esperaba el día del turno.

Algunos usuarios evalúan que la incorporación de nuevos pacientes podría eventualmente redundar negativamente en la calidad de la atención. Así, un entrevistado planteó su duda acerca de que los médicos podrían verse obligados a acortar el tiempo de cada sesión con el objetivo de poder atender a una mayor cantidad de pacientes. Esta observación sobre el ritmo o el "apuro" de los médicos lleva a que en muchos casos los pacientes prefirieran no hacerles preguntas sobre cómo funciona la acupuntura o se inhiban de iniciar una conversación para no "sacarles" el escaso tiempo del que disponen:

\footnotetext{
“No se puede [preguntar más] porque tienen tantos pacientes y tienen que ir rápido, no se pueden quedar a explicar al paciente [...]. No me da para decirle "¿doctor me podría explicar esto?" Porque él tiene muchos pacientes y el tiempo es realmente importante aquí". (E07)
}

La aceleración del ritmo de la atención va en detrimento del trato más personalizado que los pacientes esperan, y que los mismos médicos querrían brindar. En este sentido, uno de los médicos refirió a la necesidad de "ralentizarlo" para alcanzar dicha meta en la atención.

La posibilidad de no poder elegir al médico que realizará el tratamiento también fue observado por una entrevistada, pero lo justifica por ser un ámbito público, "para elegir el profesional tenés que ir al consultorio y pagar la consulta" (E09).

Para colaborar en la superación de las restricciones existentes en el servicio cuando realizamos el trabajo de campo varios pacientes estaban organizando una cooperadora para reunir fondos y movilizándose con el objetivo de solicitar a las autoridades del hospital la asignación de mayores recursos al servicio. Además de esta iniciativa, algunos entrevistados plantearon la necesidad de incorporar más servicios de acupuntura en otros hospitales y en los centros de salud comunitarios para descentralizar la oferta, favorecer la accesibilidad, y descomprimir de este modo la sobredemanda de tratamientos que observaban en el servicio:

\footnotetext{
"Si pudiera cambiar, que haya en otro lugar, que pongan en otro lugar para que así no sea tan pesado para ellos [los médicos] que los horarios a veces no saben cómo van a acomodarlos con los turnos, que hubiese en otro lugar, en otro hospital". (E15)
}

\section{Discusión}

La oferta gratuita de acupuntura hace accesible a amplios sectores de la población una medicina no convencional de probada eficacia clínica para varias dolencias crónicas. En este sentido, los usuarios de un servicio hospitalario de acupuntura son "atípicos", ya que utilizan una MAC a la que en el contexto argentino acceden mayoritariamente sectores medios y altos que pueden pagarla en el sector privado. 
Los pacientes que entrevistamos incorporan la acupuntura en sus prácticas de cuidado de la salud - que en muchos casos además de la biomedicina incluye otras MACs, medicinas tradicionales y religiosas, y prácticas de autocuidado - porque la encuentran eficaz y beneficiosa (en múltiples sentidos) y porque les permite eliminar y/o reducir la ingesta de fármacos. No sólo el costo económico del cuidado disminuye sino también los efectos adversos que pueden ocasionar algunos fármacos en el tratamiento de dolencias crónicas. La literatura internacional señala que el uso de MACs expresa una insatisfacción con distintos aspectos de la biomedicina, especialmente para el tratamiento de padecimientos crónicos (Pound et al., 2005; Barnes et al., 2004; Sharma, 1996). Nuestro trabajo indica que los usuarios de acupuntura se resisten a aceptar tratamientos convencionales de limitada eficacia y que les resultan perjudiciales por sus efectos iatrogénicos, o que buscan una solución menos agresiva frente a intervenciones quirúrgicas menores cuando otro camino terapéutico les es accesible. Esta búsqueda expresa el deseo de ejercer un mayor control sobre sus cuerpos y el cuidado de su salud.

Un resultado particularmente interesante del estudio es que para los usuarios, el servicio de acupuntura no solamente funciona como un ámbito médico en donde se les brinda una solución para su problema de salud sino también como un espacio de aprendizaje y empoderamiento para ejercer un rol más activo cuando interactúan con los médicos convencionales. Varios usuarios encuentran en los profesionales del servicio la guía y el asesoramiento que les permite percibirse con un mayor margen de maniobra cuando recurren a la medicina convencional, sea para "negociar" tratamientos o para ser más demandantes en cuanto a la atención que se les brinda.

En cuanto a las diferencias positivas respecto de la atención en otros servicios hospitalarios, y de obras sociales y del PAMI, los entrevistados señalan la organización eficiente, el respecto y cuidado con los que son atendidos, las características personales de los médicos - en especial el compromiso y la pasión por su trabajo - y la buena disposición del personal administrativo. Pese a las múltiples restricciones de personal y espacio con las que funciona el servicio, los usuarios observan que existe un esfuerzo coordinado para ofrecer una atención de calidad y humanizada. Este esfuerzo redunda positivamente en la continuidad de los tratamientos.

Los entrevistados resaltan cómo ha crecido la demanda de tratamientos en el servicio en los últimos años y su impacto en un contexto institucional de escasez de recursos humanos y materiales. Señalan lo limitado de los horarios de atención, la necesidad de contar con más profesionales rentados, y el insuficiente espacio como condicionantes para satisfacer dicha demanda. Por un lado, justifican los problemas de su funcionamiento (demora en el acceso al primer turno, poco disponibilidad de tiempo para con cada paciente) por ser un ámbito público. Incluso se autoregulan para no "abusar" en la solicitud de turnos o en el "uso" del tiempo de los médicos durante las sesiones. Pero, por el otro, también advierten que estos factores afectan la calidad de la atención, especialmente la disponibilidad de turnos y la interacción médico-paciente, y esperan que la sobredemanda pueda descomprimirse asignando más recursos al servicio y mediante la provisión de tratamientos en otros hospitales y centros comunitarios.

Esperamos que nuestro estudio contribuya a hacer visible una experiencia de medicina integrativa en el sector público que ha logrado sostenerse en el tiempo gracias al esfuerzo de los profesionales, la gran demanda de los usuarios que encuentran una solución satisfactoria y accesible materialmente para sus problemas de salud, y el apoyo de las autoridades sanitarias de la provincia. Que las MACS sean accesibles para los sectores menos privilegiados dependerá de políticas de salud que permitan y garanticen que estas experiencias de integración dejen de ser una excepción, cuenten con mayores recursos financieros y edilicios, y se difundan en todo el país en el ámbito público. 


\section{Colaboradores}

Las autoras Betina Freidin y Rosana Abrutzky elaboraron el artículo de manera conjunta. Betina Freidin realizó las entrevistas en el Servicio Hospitalario en el que se llevó a cabo el estudio, y realizó las revisiones del texto.

\section{Agradecimientos}

La primera autora agradece muy especialmente a los usuarios, al director, a los médicos, y al personal administrativo del servicio de acupuntura por su participación en el estudio, interés en el mismo, y generosa disposición para realizar las entrevistas y observaciones. Ambas autoras agradecen los comentarios y sugerencias de los evaluadores anónimos durante el proceso de referato del manuscrito.

\section{Referencias}

ASTIN, J.A. Why patients use alternative medicine. JAMA, v.279, n.19, p.1548-53, 1998.

BARNES, P.M.; BLOOM, B.; NAHIN, R.L. Complementary and alternative use among adults and children: United States, 2007. Hyattsville: National Center of Health Statistics, 2008. (National Health Statistics Reports, n.12).

BARNES, P.M. et al. Complementary and alternative medicine use among adults: United States, 2002. Hyattsville: National Center for Health Statistics, 2004. (Advance data from vital and health statistics, n.343).

BIANCONI, Z.M.A. et al. ¿Qué dicen los pacientes? Un estudio de las comunicaciones expresadas a través del buzón de sugerencias. Med. Soc., v.23, n.4, p.186-192, 2000.

CAROZZI, M.J. Nueva era y terapias alternativas. Buenos Aires: Universidad Católica Argentina, 2000.

CERRUTTI, M.; FREIDIN, B. Evaluando la calidad de la atención en servicios de planificación familiar en la ciudad de Buenos Aires: medidas estandarizadas y percepción de las usuarias. Buenos Aires: CENEP, 2004.

FREIDIN, B.; ABRUTZKY, R. Transitando los mundos terapéuticos de la acupuntura en Buenos Aires: perspectivas y experiencias de los usuarios. Buenos Aires: Instituto de Investigaciones Gino Germani, Universidad de Buenos Aires, 2010. (Documento de Trabajo, 54).

IDOYAGA MOLINA, A. Cultura, enfermedades y medicinas: reflexiones sobre la atención de la salud en contextos interculturales de Argentina. Buenos Aires: Instituto Universitario Nacional del Arte, 2002.

IDOYAGA MOLINA, A.; LUXARDO, N. Medicinas no convencionales en cáncer. Medicina, v.65, p.390-4, 2005.

LLOVET, J.J. Servicios de salud y sectores populares. Buenos Aires: CEDES, 1984.

ORGANIZACIÓN PANAMERICANA DE LA SALUD. Informe del taller de medicinas y terapias tradicionales, complementarias y alternativas en las Américas: políticas, planes y programas. Cuidad de Guatemala, 19 y 20 de marzo. Washington: Organización Panamericana de la Salud, 2002.

POUND, P. et al. Resisting medicines: a synthesis of qualitative studies of medicine taking. Soc. Sci. Med., v.61, n.1, p.133-55, 2005. 
RAMOS, S.; PANTELIDES, E.A. Dificultades en la prevención secundaria del cáncer de cuello de útero: las mujeres y los médicos frente a una citología cervical positiva. Buenos Aires: CEDES, 1990. (Documentos CEDES, 43).

SHARMA, U. Using complementary therapies: a challenge to orthodox medicine?. En: WILLIAMS, S.Y.; CALNAN, M. (Orgs.). Modern medicine: lay perspectives and experiences. Londres: UCL Press, 1996. p.230-55.

VITURRO, M. Complementary alternatives in medicine: the case of a religious healer in Buenos Aires, Argentina. Scripta Ethnol., v.20, p.129-41, 1998.

WORLD HEALTH ORGANIZATION - WHO. Global Atlas of traditional, complementary and alternative medicine. In: ONG, C.K. et al. (Orgs.). Geneva: WHO Press, 2005. p.51-5.

FREIDIN, B.; ABRUTZKY, R. Acupuntura em um serviço hospitalar na Argentina: experiências e perspectivas dos usuários. Interface - Comunic., Saude, Educ., v.15, n.37, p.505-18, abr./jun. 2011.

Investigamos experiências e perspectivas dos usuários de um serviço hospitalar público de acupuntura do noroeste argentino. Baseamos a análise em 18 entrevistas semiestruturadas realizadas no ano 2005. Os entrevistados recorrem à acupuntura por doenças crônicas, especialmente como paliativo da dor, embora vários estendam seu uso para outros problemas de saúde, diante da ineficácia e/ou efeitos adversos dos tratamentos biomédicos, ou para evitar cirurgias menores. Incorporam a acupuntura a estratégias pluralistas de cuidado, que junto com a biomedicina incluem tipicamente outras medicinas não convencionais. O contexto hospitalar facilita o fato de experimentar uma medicina alternativa. Vários usuários buscam o serviço de apoio para "negociar" os tratamentos com seus médicos convencionais. Destacam a eficiência organizacional do serviço e o compromisso dos médicos. Diante da demanda crescente de atenção em um contexto de escassez de recursos os usuários advertem que ela pode afetar a disponibilidade para marcar consultas médicas e a interação médico-paciente.

Palavras-chave: Medicina alternativa e complementar. Integração. Acupuntura. Serviços de saúde públicos. 